\title{
Posttraumatic growth and psychosocial gains from adversities of korean special forces: a consensual qualitative research
}

\author{
Junsang Park ${ }^{1}$ Jungmin Lee ${ }^{2}$ (D) Daewon Kim ${ }^{1}$ Joonsuk Kim³
}

Accepted: 9 September 2021

(c) The Author(s), under exclusive licence to Springer Science+Business Media, LLC, part of Springer Nature 2021

\begin{abstract}
Because military special forces carry out dangerous missions, they are much more exposed to adversities and traumatic events compared to other occupational groups. According to Posttraumatic Growth theory, individuals tend to obtain positive growth through adversities. Moreover, a framework of Psychosocial Gains from Adversity argues not only individual changes but also social changes in the group to which the individual belongs are induced. Therefore, the present study aimed to explore the adverse experiences of special forces operatives and delineate the positive shift at an individual and social level via Consensual Qualitative Research (CQR). Eight individuals serving in special forces at the Korean Army, Navy and Air Force were interviewed using a semi-structured protocol. Four domains including 10 categories and 34 subcategories were identified: (a) Adverse experiences; (b) Personal change; (c) Social change; and (d) Attributes related to adverse experience. The findings and clinical implications are discussed in light of growth over facing adversities and interaction between personal and social factors.
\end{abstract}

Keywords Posttraumatic growth · Psychosocial gain from adversity · Special Forces · Consensual qualitative research · Military personnel

\section{Introduction}

Special Forces are representative army units of South Korea established to execute special operations. A special operation is a mission carried out regardless of war or peacetime in order to oversee emergencies or strategic contingencies. Operatives are meticulously selected according to physical

Jungmin Lee

sylphmin@gmail.com

Junsang Park

earth_js@snu.ac.kr

Daewon Kim

kimdaewon327@snu.ac.kr

Joonsuk Kim

sneaker112@snu.ac.kr

1 Department of Education, Seoul National University, Gwanak-ro 1, Gwanak-gu, Seoul, South Korea

2 Hannam University, Hannam-ro 70, Daedeok-gu, Daejeon, South Korea

3 The Catholic University of Korea, Eunpyeong St. Mary's Hospital, 1021, Tongil-ro, Eunpyeong-gu, Seoul, South Korea assessment and aptitude test, then go through intense military training requiring extreme physical fitness. The Special Forces of Korea was founded on the 25th of February, 1950 with the breakout of the Korean war. It has since been divided into Army Special Warfare Command, Underwater Demolition Team/Sea Air Land (UDT/SEAL), Marine Corps Special Reconnaissance Unit (MCSRU), Special Air Force Rescue Team (SART) and so on based on the nature of the operations required by different armed forces (Special Warfare Command, 2016). They are also progressing to become even further specialized in line with modern warfare environment.

Special force operatives must conduct specialized missions under more extreme circumstances in contrast to regular soldiers. Such examples demonstrating highly intense operations would be conveying reconnaissance missions in the middle of enemy grounds with minimal resources or recovering to base after an engagement. Because of the demanding job facets, special force operatives frequently experience adversities, predisposing them to poor mental health such as heightened risk of post-traumatic stress disorder (PTSD) (Chappelle et al., 2014; Morrow et al., 2013; Ogle \& Young, 2016). For instance, $11.6 \%$ of American 
Air Force pararescuemen are diagnosed of PTSD, 1.6\% of depression and $16.1 \%$ with insomnia (Morrow et al., 2013). Furthermore, in a study of US Air Force Special Tactics operators who have experienced being stationed in countries such as Afghanistan, Iraq, and Syria, 26\% of the crew were found to be going through high levels of stress and anxiety (Ogle \& Young, 2016). Military units carrying out special missions in general also report increased stress levels or burnout (Chappelle et al., 2014). As such, studies indicate special forces groups are prone to experiencing physical/ psychological hardships during highly intense training and dangerous missions.

Since adversity has been considered to cause psychological distress, many studies have focused on clarifying its negative influence or therapeutic interventions. However, not only overcoming the influence of an adversity but experiencing growth in the process is gradually receiving attention. Tedeschi and Calhoun (1996) first introduced the term posttraumatic growth (PTG) suggesting "people exposed to even the most traumatic events may perceive at least some good emerging from their struggle with such tragedies" and pioneered its research. PTG studies in soldiers have also showed that although adversities can induce stress and negative emotions, it can also promote resilience (Jackson et al., 2012; Sudom et al, 2014), life satisfaction (Feder et al., 2008; Morgan et al., 2017), mental functioning (Greenberg et al., 2021; Tsai et al., 2015), and spirituality (Feder et al., 2008).

Tedeschi and Calhoun (2004) analyzed previous studies and categorized PTG into 5 areas: 1) more appreciation of life and changes in values, 2) developing closer and more meaningful relationships, 3) perceiving better personal strength, 4) becoming aware of new possibilities and opportunities in life, and 5) more contemplation in fundamental existential questions. Qualitative PTG literature in soldiers confirms this in the context of military environments, such as appreciating life itself (Larick \& Graf, 2012; Palmer et al., 2017), empathizing more (Scott et al., 2011), and being more accepting towards change (Palmer et al., 2017). Qualitative PTG research also confirmed changes in social relationships, such as a better connection with friends and family (Palmer et. al., 2017; Wick \& Goff, 2014) or increased sense of belonging and fellowship in one's society (Larik \& Graf, 2012; Scott et al., 2011).

Although PTG can be moderated by a number of variables, recent studies regarding soldiers consistently emphasize the importance of a secure social support system in facilitating PTG (Mark et al., 2018). Soldiers with stronger social network (Tsai et al., 2015), better relationship with teammates (Pietrzak et al., 2010) or healthier relationships as a couple or with family members (Forstmeier et al., 2009; Palmer et al., 2017; Wick \& Goff, 2014) experienced more PTG. It can indeed be inferred that PTG is a social process as much as a personal one, in which the individual going through the adversity requires support from close members and the community. Therefore, in order to understand the experience of growth after an adversity, it is noteworthy to explore what social support/systematic factors individuals experience during the adversity and growth.

The dominant theories concerning growth following adversities are post-traumatic growth, benefit-finding, adversarial growth, and stress-related growth, all focusing on intrapersonal change (Linley \& Joseph, 2004; Park et al., 1996; Tedeschi \& Calhoun, 2004). Recently Mancini (2019) highlighted the social influence that functions as a protective factor and promotes growth, naming the term psychosocial gains from adversity (PGA). PGA is a social environment which protects individuals from further traumatic events, and is formed when a community as a whole suffers from a traumatic occurrence such as natural disasters or terrorism. The group experiencing the incident receives meaningful support from their social environment (Lakey \& Orehek, 2011) and the group members develop trusting relationships, more collaboration, group identity, social interactions, practical conversations, and prosocial actions. This forms a prosocial environment, leading to PGA. An important point to consider is that PGA can help understand individual patterns in reacting to acute stress (Mancini, 2019). This is because while PTG mainly focuses on growth gained by contemplation after the traumatic event, PGA concentrates on the immediate responses of individuals and the group. However, despite such implications, PGA has not been looked into enough (Andersen et al., 2014; Dickstein et al., 2010). For instance, even though marked improvement in deployment stress of U.S. soldiers could possibly be a result of 'unit cohesion' at the deployment site, the subject has been relatively neglected in the related body of research (Mancini et al., 2016).

Former studies suggest roughly four mechanisms in how PGA is driven (Mancini, 2019). An adversity stimulates automatic social-affiliative and prosocial behavior (Morgan et al., 2011; Putnam, 2002), substantively increases meaningful social interactions (Abrams et al., 2004), promotes a cooperative prosocial environment (Didion, 2007; Kaniasty \& Norris, 2004), and lastly benefits psychological functioning (Diener \& Seligman, 2002; Lakey \& Orehek, 2011). Such mechanisms may be well applied to special forces groups since they act as a team in all situations. This team culture can have a positive influence on operatives adjusting to and overcoming adversities. Soldiers in general are required to cooperate with each other relatively more than other professions, which can be a catalyst for PGA. Studies demonstrating how social support and morale was boosted after deployment (Maguen \& Litz, 2006), or how unit cohesion and morale was related to lower onset of PTSD symptoms (Dickstein et al., 2010) supports the idea. Mitchell et al. (2013) witnessed that strong unit cohesion six months after 
deployment in a foreign country was positively correlated to PTG, and Mouthaan et al. (2005) also found tighter bonds among soldiers through deployment in foreign nations.

In stressful situations team members will become more cohesive and interact more with each other, which can lead to better performance as well as subjective well-being. In this perspective, not only the personal factors (PTG) of a special force operative but social influence (PGA) should be examined as well. In other words, factors such as a sense of belonging, meaningful interactions between colleagues, or an atmosphere giving spirit to the team could induce PTG among special forces operatives. However, since research is sparse regarding PGA of soldiers, the current study chose special forces operatives, who have a high probability of going through adversities, as participants. Exploring the personal growth or social benefits that these individuals experienced after a traumatic experience would contribute in preventing negative effects and invigorating positive changes. Therefore, the current study aims to explore both personal changes (PTG) and social influence (PGA) for a richer and fuller context of the subjective experience of special forces operatives as they pass through adverse events.

In this study, we aim to research PTG and PGA of special forces operatives who have experienced adversities utilizing qualitative methods. Due to a lack of studies focusing on the adversities of special forces operatives, and the experience of adversities and accompanying change are diverse and specific to each individual, a qualitative approach was preferred to investigate the context and contents of the operatives' PTG and PGA (Habib et al., 2018). The interviews were analyzed through consensual qualitative research (CQR). $\mathrm{CQR}$ is a well established qualitative method that explores phenomenological contents with high level of objectivity and validity (Hill, 2012). CQR is mainly purposed to extract domains (e.g., psychological adversity) and core idea (e.g., pathological mental symptoms) based on research team's consensus. We aim to examine what the special forces operatives perceived as a traumatic event, what the perceived personal and social changes were after the event, and what the implications are in providing a safer environment during training and carrying out missions. Moreover, we infer personal and social factors for non-soldiers' post-traumatic recovery, growth and adaptation.

\section{Method}

\section{Participants}

A typically adequate number of participants for $\mathrm{CQR}$ ranges from 3 to 15 people (Hill, 2012), so a total of eight special forces operatives were recruited, all who have served in units which take on special missions under the Korean Ministry of National Defense (Table 1). Among potential participants, only those who answered to have had experienced adversities during their career were selected. Participants included two operatives from Underwater Demolition Team/Sea Air Land (UDT/SEAL), two from Marine Corps Reconnaissance Battalion (MCRB), two from Special Air force Rescue Team (SART), and two from ROK Army Special Warfare Command (SWC). Participants were deliberately recruited from different groups to cover various special forces experiences. All participants are males, ranging in age from 26 to 32 $(M=28.50, S D=2.78)$, and service years from 2 to 9 years $(M=3.88, S D=2.23)$.

\section{Research Team}

In this study, an interview and research team of four appraisers ( 1 female, 3 males; 4 Asian; Age $M=31 S D=9.38$ ) and one supervisor participated in the analysis. The consensual team consisted of a doctor in educational counseling who had an experience in qualitative research methods and PTSD research, with a career as a certified counselor for more than 10 years. The other team members were three graduate students; one majoring in clinical psychology and two in
Table 1 Demographic characteristics of the eight participants

\begin{tabular}{llllllll}
\hline Case & Affiliation & Age & Gender & Rank & $\begin{array}{l}\text { Years } \\
\text { of ser- } \\
\text { vice }\end{array}$ & Marital Status & Active/Reserve \\
\hline 1 & UDT/SEAL & 25 & M & Navy chief petty officer & 4 & Single & Active \\
2 & UDT/SEAL & 24 & M & Navy petty officer 1st Class & 4 & Single & Active \\
3 & ROK-SWC & 26 & M & First Lieutenant & 3 & Single & Reserve \\
4 & SWC & 30 & M & Sergeant First Class & 9 & Single & Active \\
5 & MCRB & 27 & M & Lieutenant & 2 & Single & Reserve \\
6 & SART & 31 & M & Technical Sergeant & 3 & Married & Active \\
7 & SART & 31 & M & Technical Sergeant & 4 & Married & Active \\
8 & MCRB & 26 & M & Lieutenant & 2 & Single & Reserve \\
\hline
\end{tabular}

UDT/SEAL $=$ Underwater Demolition Team $/$ Sea Air Land, MCRB $=$ Marine Corps Reconnaissance Battalion, $\mathrm{SART}=$ Special Air force Rescue Team, SWC $=$ Army Special Warfare Command 
educational counseling, all who served the Korean Army. The graduate students had completed coursework and had at least one year of experience in counseling. The supervisor was a counseling faculty with more than 30 years of counseling experience and a number of research experience including $\mathrm{CQR}$ research.

Prior to analyzing the data, research team members discussed biases, stereotypes and expectations. The purpose of the discussion was to minimize their influence to the analysis procedure to endorse objectivity (Hill et al., 1997, 2005). There was an agreement that it would be difficult for special forces operatives to recognize and express their difficulties or psychological conditions since the organization would have an atmosphere that may pressure members to mask their weakness and suppress help-seeking behaviors. It was also confirmed that team members had expectations for positive changes after an adversity, therefore agreed to be cautious and aware so that such bias would not affect the interview or analysis process.

\section{Data Collection Process}

Data was collected for four months from December 2019 to March 2020. During a preparatory stage the research team organized semi-structured questions based on existing adversity-related studies. The questions were further revised after consultation with the team's supervisor. Introductory interview questions included demographics, satisfaction with current duties, reasons for the current career choice, and performed tasks. Main questions were divided into personal and social changes after the adverse experience (Table 2). In order to explore the adversities related to being a special force operative, the interview focused on participants' physical and psychological experiences such as training, work hours, interpersonal relationships within work and overseas dispatch.

Purposive sampling was used since the goal of this study was to explore in-depth the experience of subjects exposed to a relatively specific task environment. Purposive sampling method is the process of subjectively selecting samples considered suitable for research, which may be influenced by the bias of researchers but is efficient and suitable for qualitative studies investigating a particular group. Two UDT/SEAL members, three ROK-SWC members, and two ROK-MCRB members were recruited through an acquaintance of a research team member. Two SART members were additionally recruited through online social networking services. Since one SWC member was prohibited to contact civilians due to policies of his troop during the COVID-19 pandemic, a total of eight people were interviewed. Before the process, participants were asked if they had experienced adversities during their service, and only if they did were they considered as candidates.

Interview procedures followed the method recommended by Hill (2012). Before the interview participants were first introduced to the process and gave agreement to participate. The research team then sent interview questions a week before the interview so that participants could prepare. On the day of the interview, the research team received additional written consent for participation to the study and conducted a semi-structured interview. The interviews lasted from 90 to $120 \mathrm{~min}$, were all recorded, and were conducted by the researchers themselves in an enclosed individual space near the participants' military units or residences. The recorded files were transcribed, after which all personal information were deleted and given unique case numbers.

Table 2 Interview questionnaire

\begin{tabular}{|c|c|}
\hline Topic & Question \\
\hline Adverse experience & $\begin{array}{l}\text { When working as a special forces operative, were there events of adversities that included acute stress, extreme physical } \\
\text { or psychological pain? } \\
\text { Tell us in detail the adversity that you experienced }\end{array}$ \\
\hline Personal Change & $\begin{array}{l}\text { Have you experienced any psychological changes after the adversity? } \\
\text { After the adversity, were there changes in how you perceive yourself? Were there any changes in how you perceive the } \\
\text { world? } \\
\text { After the adversity, were there changes in how you approach interpersonal relationships? } \\
\text { After the adversity, were there changes in your philosophical understanding of life? }\end{array}$ \\
\hline Psychosocial Change & $\begin{array}{l}\text { After the adversity, were there changes in interpersonal relationships? } \\
\text { After the adversity, were there changes in thought or beliefs regarding your family, friends or colleagues? } \\
\text { After the adversity, were there changes in how you cooperate with others or help each other to accomplish a task? } \\
\text { After the adversity, were there changes in thoughts or beliefs regarding the team or group you serve in? } \\
\text { After the adversity, were there changes in what or how you communicate with your family, friends or colleagues? }\end{array}$ \\
\hline
\end{tabular}




\section{Data Analysis Process}

Analysis was conducted using consensual qualitative research $(\mathrm{CQR}) . \mathrm{CQR}$ is a method that allows the research team to examine individual experience and attitudes, or uncommon incidents in depth (Hill, 2012). In order to review details of each special operative's phenomenological experience and analyze the personal, social changes they went through, CQR was a good fit as a means to approach the topic. Data analysis included the process of extracting domains and core categories along with cross analysis to reach a consensus in categorization. Lastly, domains were labelled based on how frequent it was discovered within the data. Analysis results were revised according to feedback from the supervisor.

Coding into Domains and core ideas Research members first independently developed domains by reading through the first two cases. Then the domains were revised through extensive discussion until a consensus was reached concerning the list of domains. These domains were used to code all cases, being revised as any new data emerged which were unclassified. The coding process was repeated every time the domains were altered. In all meetings, the speaking order was altered to hinder any team member becoming too dominant. Finally, four main domains were extracted: Adverse experience, Personal change, Social change, and Characteristics related to overcoming the adversity. After mapping out the domains, the research team formulated core ideas based on the domains. Upon establishing the domains, the core ideas were only merged in with members' consensus.

Auditing of Domains and Core Ideas After coding all data $(\mathrm{N}=8)$ and constructing core ideas (i.e., abstracts in fewer or more succinct terms) in each domain, an external auditor who is a CQR professional supervised the results. The supervisor examined each domain and core idea separately, giving feedback to balance out their specificity or abstractness. The domains and core ideas were revised accordingly.

Cross-Analysis Research members independently created categories that reflected the themes present in each domain's core ideas. Then after extensive discussions, both offline and online, the team came to a consensus with a final list of categories. Based on recommendation of previous studies such as Hill et al. (2005), a category which appeared in all cases $(\mathrm{N}=8)$ was considered general, more than $50 \%(\mathrm{~N}=4 \sim 7)$ typical, and less than $50 \%(\mathrm{~N}=2 \sim 3)$ variant. Categories reported only once were excluded from the analysis. Analysis results were revised after consulting with the supervisor in whether each domain was adequately placed in a core category, the levels of hierarchy were adequate and consistent, and whether positive perspectives should be taken into account. These results were then reviewed by interviewees if any data we used were out of context, and consent was received to use and publish the data they provided in the way it was analysed.

\section{Results}

A total of 4 domains, 10 categories, and 34 subcategories were extracted upon analysis. Domains are presented in Table 3 and its meanings are presented based on the original transcript.

\section{Adverse Experience}

Participants reported various adverse experiences, mainly in training, physical exercise, heavy workload, and stress in workplace relationships. These experiences can largely be divided into physical and psychological adverse categories. Physical adversities consisted of one subcategory, yet appeared in all cases. In psychological adversities, 'negative emotions due to adversities' was typical while other subcategories were variant, which demonstrated the individual differences in causes and symptoms of psychological adversities between participants.

\section{Physical Adversity}

Participants reported physical fatigue from withstanding extreme conditions such as sub-zero temperature, starvation, $132 \mathrm{~h}$ sleep deprivation training and $400 \mathrm{~km}$ march course. Undergoing extreme training resulted in participants passing out and being injured.

"It's like I was really going to die because training was so exhausting. It's a beginner level training, but they don't give you any water or food, and you can't sleep for 132 hours. It's just endless training...” (case 1) "The training comes to an end, as long as you hang in there and don't give up. In the end you find yourself stronger. One of my teammates kept passing out every time he tried to swim $50 \mathrm{~m}$ underwater. Passing out doesn't happen often, but you need to hold your breath until you're literally on the brink of passing out." (case 2)

\section{Psychological Adversity}

Categories in psychological adversity were negative emotions from adversity, pathological mental symptoms, and intense interpersonal stress during adverse events. Special 
Table 3 Categories, category, subcategory related to special forces' adverse experience

\begin{tabular}{|c|c|c|c|}
\hline Domain & Category & Subcategory & Frequency \\
\hline \multirow[t]{8}{*}{ Adverse experience } & Physical adversity & Extreme physical fatigue due to physical training and intensive training & General(8) \\
\hline & \multirow[t]{7}{*}{ Psychological adversity } & Negative emotions due to adversity & Typical(4) \\
\hline & & Pathological mental symptoms & Variant(3) \\
\hline & & Intense interpersonal stress & Variant(3) \\
\hline & & Mental stress due to excessive work & Variant (2) \\
\hline & & Mental stress in the situation of being dispatched & Variant $(2)$ \\
\hline & & Burnout & Variant $(2)$ \\
\hline & & Stress due to high responsibility & Variant (2) \\
\hline \multirow{10}{*}{$\begin{array}{l}\text { Personal } \\
\text { change }\end{array}$} & \multirow[t]{3}{*}{ Change in values } & Can-do spirit & Typical(4) \\
\hline & & Challenging spirit & $\operatorname{Variant}(2)$ \\
\hline & & Having a positive perspective & Variant (2) \\
\hline & \multirow{2}{*}{$\begin{array}{l}\text { Organizational and occupa- } \\
\text { tional changes }\end{array}$} & Having pride and affection for the organization & Typical(4) \\
\hline & & Increased professional competence & Variant(3) \\
\hline & \multirow[t]{3}{*}{ Personality change } & Improved confidence & Variant (2) \\
\hline & & Strengthened responsibility & Variant(2) \\
\hline & & Understanding oneself better & Variant (2) \\
\hline & \multirow[t]{2}{*}{ Interpersonal change } & Better sympathizing with others' difficulties & Variant (2) \\
\hline & & More efficiently resolving interpersonal conflicts & Variant (2) \\
\hline \multirow{8}{*}{$\begin{array}{l}\text { Social } \\
\text { change }\end{array}$} & \multirow[t]{7}{*}{ Positive social change } & Being recognized and supported by colleagues and family members & Typical(6) \\
\hline & & Enduring difficulties by uniting with each other & Typical(5) \\
\hline & & Exchanging practical help between colleagues & Typical(5) \\
\hline & & Forming a sense of bonding & Typical(5) \\
\hline & & Forming brotherhood and trust & Typical(4) \\
\hline & & Sharing difficulties, giving advice and support & Typical(4) \\
\hline & & Growth by modeling each other & Variant(3) \\
\hline & Negative social change & Becoming harsh and inconsiderate to peers & Variant $(3)$ \\
\hline \multirow{8}{*}{$\begin{array}{l}\text { Attributes related to } \\
\text { adverse experience }\end{array}$} & \multirow[t]{4}{*}{ Personal factors } & Lack of self-disclosure regarding hardships & Typical(5) \\
\hline & & Hardiness & Typical(5) \\
\hline & & Challenging spirit & Variant(3) \\
\hline & & High responsibility & Variant (2) \\
\hline & \multirow[t]{4}{*}{ Environmental Factors } & Emphasizing hardiness & Typical(4) \\
\hline & & Horizontal organizational culture & Variant(3) \\
\hline & & Atmosphere of acknowledging each other's hardships & Variant (2) \\
\hline & & Openly sharing opinions & Variant (2) \\
\hline
\end{tabular}

forces operatives experienced excessive stress during tough training that was intended to drive them into an extreme psychological state in order to improve their mental resilience. They went through negative emotions such as fear of training and shame when they failed to complete the training course successfully. They also developed dissociative symptoms and hallucinations due to physical pain and sleep deprivation.

"For example, I can't sleep for about 120 hours in the middle (of training). They don't let us sleep. If someone closes his eyes to doze off, he gets punished. ...... So I had this hallucination at some point that I was home. My mom opened the door and told me to come in, my sister was there too, and I was about to take off my clothes, take a shower and wash up. During the hallucination I was walking but I didn't realize that I was in training, I thought I was walking around home. And when I came to my senses I finally realized that it was just a hallucination." (case 5)

Special forces operatives experienced stress not only from extreme physical and psychological environments but also interpersonal relationships. There were cases of supporting and helping each other during the adversities, but in others, individuals became self-centered resulting in conflicts 
between superiors and subordinates. In addition, variant subcategories included experiencing great mental stress due to excessive workload or being dispatched to another country, excessive stress due to high responsibility or burnout after training and work. In the case of experiencing burnout, the participants went through falling into a slump, binge eating, lethargy, and losing motivation to serve, thinking of retiring.

"People become really vicious in extreme situations. Their selfish nature is revealed. So it's really hard, because of conflicts between people." (case 1)

"My mind went totally blank, for about two months after the training. I didn't want to do anything. I felt like a completely dry rag, like someone had used a machine instead of using hands. The rag stands for my willingness, and the water that's supposed to be soaking the rag was my motivation." (case 5)

\section{Personal Change}

In the domain of personal change, 4 categories and 10 subcategories were extracted. 2 subcategories were typical and the other 8 subcategories were variant, which means special forces operatives experienced diverse personal changes.

\section{Change in Values}

First, within changes in values, it was reported that through the adverse experience, participants gained a 'Can-do spirit'- a challenging and positive attitude. Half of the participants had come to believe that they can overcome anything through experiencing and overcoming physical and psychological adversities. Statement of Case 1, "Even if you think you'll die in the process, you can actually go through it if you try", "I won't die no matter how hurt and torn apart you are, I can do it if I just give it a try", and that of case 5, "I used to feel scared and burdened, but now I believe with enough time I can do anything", "I can do it because I know I'm not weak." are good examples illustrating this change. In addition, adversities greatly influenced each individual's values, such as case 2 who continued to progress towards his challenging goal despite wanting to give up, and case 5 who learned to see difficult situations in a more positive perspective rather than complaining. Case 3 in particular interpreted his adverse event positively. His statement, "It was nice to have so many special experiences....... Yes it was very hard in the process, but it was a great experience in the end." is a good example of PTG.

"It's difficult. Very, very difficult. I thought of giving up hundreds of times. But looking back on what I went through...... if there's something I want to do I have to do it anyways. The answer is already there. I can't not do it." (case 2)

"I ended up not using the word "hard". If you use the expression "it's hard for me", you weaken yourself and you're admitting that you're weak. Instead I say "it's not easy"....... There may be individual differences, but I try to be positive in such situations. Because if you don't you can't hold on." (case 5)

\section{Organizational and Occupational Changes}

Half of the participants reported they gained great pride as a member of their group and affection for their organization after experiencing adversities. Not only their personal growth but also the recognition and respect from the people around them contributed to taking pride in their organizations. Case 1 reported about this dignity, saying "After the dispatch I felt indifferent because it was natural for special forces to be sent overseas. But people around me recognized me more.......It also made my life as a special forces operative a bit more enjoyable ". Case 7 also stated, "I'm a special forces operative of the Air Force. So, there is more to talk about, and the Air Force members are proud of us too, the rescue team," expressing pride originating from Air Force members respecting the rescue team. Case 1 who reported his experience of being dispatched as an adversity, reported gaining a lot of pride through the experience. Case 6 referred to a sense of belonging as a UDT/SEAL member, especially during events when he was with other military groups. He also mentioned the UDT/SEAL beret as an insignia which is given only after completing immense training. Affection for their organization beyond pride as a member was most evident in Case 2, while Case 3 reported that the adversity made him not only feel pride and affection for his organization, but also more professionally competent.

"Being dispatched involves an anti-terrorism operation. I can improve my expertise in such jobs because you train and shoot every day. And I think it led me to have a lot of pride in myself." (case 1)

"I'm going to get discharged from here in February and I think I'll shed a lot of tears. In the 24 years of my whole life, this is where I poured all my passion and enthusiasm into. I served where such people were my colleagues....... I love my unit, UDT so much. I have so much pride in it." (case 2)

\section{Personality and Interpersonal Changes}

Subcategories in personality changes and interpersonal changes are all variant, each appearing in two cases. Personality changes reported by participants showed subcategories such as building confidence, strengthening responsibility, 
and better self-understanding. In the case of better selfunderstanding, case 2 reported that being dispatched gave him an insight of how open and free-spirited himself was, while case 8 reported that through his experience as a platoon leader he was able to understand and lay down his inferiority complex originating from his relatively small build.

In interpersonal changes, there were cases where people could better sympathize with other people's difficulties, and resolve interpersonal conflicts more effectively. The fact that participants gained personal growth regarding interpersonal relationships in a relatively inflexible military culture is particularly notable. Experiences of better understanding the difficulties of team members helped the participants to be more sympathetic to others. Effective resolution of interpersonal conflicts included an increase in the ability to communicate issues that members did not share before. For instance, case 3 reported an experience of talking to his captain, saying "I knocked on his door, a bundle of nerves. I explained my situation first, this and that, then I told him how I was disappointed in some matters and the captain was cool about that. The conversation ended well. He took care of work in a better way."

\section{Social Changes}

Because subcategories of social changes are more typical compared to personal changes, they were more consistently reported among participants. Seven subcategories were included in positive relationship changes while one was included in negative relationship changes. All subcategories except for one were typical in positive relationship changes. The subcategory in negative relationship changes was varied.

\section{Positive Relationship Changes}

The most frequently reported change was being recognized and supported by colleagues and family members. Participants reported that support of parents or colleagues was helpful in overcoming adversities and after the adversity they received recognition from people around them. For instance, case 8 reported that he went through severe stress and anxiety when he was first placed as an officer, which was reduced owing to the support of friends, colleagues and family members. A notable aspect is that because special forces operatives mostly did not expect their family members or spouse to understand what sort of difficulties they went through and also did not want to make them worry, peers were usually sought for such issues. However, case 7 reported that because his father-in-law worked as a non-commissioned officer in the Air Force he actively asked and understood what the participant was going through. Most participants also reported that after overcoming their adversities many people recognized their effort and respected them. Family members were proud of them for overcoming and completing their hardships, while peers credited their effort as well.

"I was really nervous at first. Being yelled at without even knowing why for over a month, feeling guilty and unqualified... But after a couple months people comforted me a lot saying 'That job is very challenging to everyone. You should be proud of yourself by just hanging in there'. And I became less afraid during training as well because I knew to what extent I was capable. I wasn't anxious anymore because I knew it wasn't my fault." (Case 8)

There were also social changes in the process of enduring and overcoming an adversity. Special forces operatives reported that uniting among peers, exchanging practical help between colleagues, sharing difficulties, giving advice and support, and modeling others for improvement were very helpful in overcoming adversities. First, when participants felt overwhelmed and wanted to quit during adverse experiences, they developed a sense of unity with their peers by encouraging one another to stay together and hold on. Also lending a hand while training or giving necessary help to each other such as teaching swimming was reported to help endure adversities in multiple cases. Participants additionally reported expressing their difficulties and seeking support and advice to group members. Modeling other people's behavior such as helping a peer or endeavoring to finish training despite passing out was also helpful.

"There was a thing where you had to spend 3 nights without sleeping. It was called 'combat experiment' where you need to, well experiment and all the important officers had to participate. At a point some people said 'what the heck let's do it', 'we can do it together' and from there we pushed ahead really well." (Case 3) "I wasn't good at sea swimming but I had to complete the $3.2 \mathrm{~km}$ course within 2 hours. I couldn't make it in time. That is why I was really thankful when people helped by pushing me from behind. Even though we were all assessed individually and it was a competition." (Case 8)

Similar to the aforementioned situations, typical changes such as a sense of bonding or brotherhood and trust also appeared as a result of positive influence on one another through social relationships. Case 1 who is a member of UDT/SEAL explained that soldiers, non-commissioned officers and officers all receive beginner level training together and as people drop out, the remainders bond more and more with each other. Case 4 also commented he is very thankful to his team members who were nice to him. Building trust as they overcame training with a high dropout rate together was typical as well. Because the job description of 
special forces groups is very dangerous and life threatening, knowing that they can trust their colleague in a mission is crucial. Case 1 reported "we really need a brother-in-arms whom we can trust to cover our back during a mission" and emphasized "The most important thing for the team is whether they can entrust their blind spots to each other". This job characteristic influenced training drills to require a lot of teamwork and contribute to building up trust.

\section{Negative Relationship Changes}

Even though positive changes were mostly reported, 3 cases reported negative changes. Seeing that adversities induced much stress, participants grew apart with certain people who tried to avoid training, became burnt-out and could not be considerate of other people. People would get more easily irritated and this led to colleagues blaming or ignoring each other in some circumstances.

\section{Characteristics Related to Overcoming Adversities}

Lastly, the fourth domain is the characteristics related to overcoming adversities and leading to positive changes, which was not the main research question but was mentioned repeatedly in various context during the interviews. It was included in the analysis because factors such as characteristics of the individual or the organization were considered meaningful in understanding how special forces operatives overcome adversities and gain personal growth. Characteristics related to overcoming adversities were categorized into personal factors of special forces operatives and environmental characteristics of which the team was situated in.

\section{Personal Factors}

Four subcategories arose as personal factors of special forces operatives, mainly being one's personality characteristics. Typical subcategories were the lack of self-disclosure regarding hardships and hardiness. Lack of self-disclosure regarding hardships was not because operatives did not want parents or close friends to be affected but because they simply did not feel the need to do so. This aspect was also related to hardiness, since some participants saw openly discussing their hardships as a sign of weakness. Participants with hardiness did not give up in strenuous situations and tried to complete their task as much as possible. This characteristic influenced them to not rely on others or give up during an adversity but to try to endure by themselves.

"So... I never told much to anybody. Just keep things to myself. This is my task and it is my responsibility. (...) I don't really express it when something really weighs down on me. Even if it's hard, it's a good experience when I eventually overcome it right?" (case 3)

Variant categories were a challenging spirit (3 cases) and high responsibility ( 2 cases). A challenging spirit was related to choosing special forces as the participants' career. High responsibility was not typical but influenced participants to endure difficult missions and training.

"Yes. I've always been a responsible person since I was little. Maybe it was because my parents always said 'you always get it done when you say so', but I feel whenever I say I would take care of something I need to do it one way or the other, or else I'd feel incompetent." (case 8)

\section{Environmental Characteristics}

Characteristics of the team that influenced how participants overcome adversities were emphasizing hardiness, a horizontal organizational culture, promoting to acknowledge each other's hardships, and openly sharing opinions for personal development. A culture that emphasizes hardiness was the only typical subcategory, showing special forces operatives had to be physically and psychologically tough in order to carry out missions. Candidates were selected based on how tough they were, which was further strengthened by breaking through their limits by training.

"An ambulance always accompanies us because it's the law but the mindset they teach us goes like this: You don't ride the ambulance unless your body parts fly away more than several feet. They teach us grit, to endure until the end.. It's something like, 'you do it until you get it done'." (case 1)

The environment surrounding Special Forces operatives not only emphasized hardiness but also credited members who completed training and overcame their limits. Also, unlike the general notion of a highly vertical organizational culture existing in the army, in order to effectively train operatives and carry out missions, a culture was formed where members shared feedback such as opinions and advice regardless of rank differences. Such horizontal organizational culture was a factor enabling operatives to improve themselves and endure a dangerous and extreme environment.

"Oh, we're not authoritative at all. Our seniors always say everybody's the same in front of a bullet......Even the newest juniors always speak out their mind to seniors, even to people who have trained over 20 years. Of course, they put it mildly but definitely deliver their opinions, like how the seniors are doing things wrong, how things should be done differently. And seniors 
always keep their ears open, so that they can improve." (case 2)

\section{Discussion}

The present study qualitatively explored the adversities of Korean special forces operatives, their personal/social changes after adversities, and characteristics that were helpful in overcoming them. Regarding physical adversities, all participants reported extreme physical fatigue due to intense training and workout routines which well underlined the characteristics of a special forces operative, that such adversities are part of their job but also a trigger of acute stress. Psychological adversities were relatively diverse compared to physical adversities. During training and undergoing missions, participants experienced trauma-related emotions, dissociation, or hallucinations. Also, interpersonal conflicts during extreme conditions incited stress, burnout and slumps. Compared to previous PTG studies which listed stress or traumatic events as bereavement, accident, disaster, physical disease, sexual abuse, or war (Linley \& Joseph, 2004), adverse experiences found in this study were different such as burnout due to extreme training and workload or interpersonal conflicts during extreme conditions. However according to Tedeschi and Calhoun (1995), traumatic events are operationally defined not by the objective features they possess but based on the subjective perceptions and negative psychological reactions accompanied by related experiences. Such definition focuses on the importance of subjective experience and individual differences in reacting to it. Furthermore, PTG theory entails the traumatic events are so challenging that it can potentially destabilize one's environment including all internal and external resources, along with the personal schema used to understand the world (Tedeschi \& Calhoun, 2004; Tedeschi \& Calhoun, 1995). In this study, all participants reported negative valences or intense psychological reactions towards the traumatic events followed by changes in personal schemas reinforcing how these experiences could be understood as adversities.

The psychological adversities Korean special forces experienced were mainly emotional and psychological symptoms caused by excessive stress during extremely intense training. This is similar to emotional symptoms individuals experience after a trauma. Individuals who have suffered traumatic events experience a variety of emotions such as a sense of betrayal, self-criticism, fear, alienation, anger, and shame (DePrince et al., 2010). Since participants reported self-criticism, fear and shame, it can be seen that the events during training and work were perceived as traumatic events. In addition, Special Forces operatives reported dissociations and visual auditory hallucinations during training which is in line with previous studies showing soldiers that undergo intense training or extreme missions experience similar brief psychotic episodes (Arens, 2015; Eid \& Morgan, 2006).

Personal changes participants experienced after the adversity were changes in values, organizational and occupational changes, personality changes, and interpersonal changes. Changes in values included a can-do spirit, challenging spirit, and a positive attitude. Participants expressed more pride and affection for their organization, and increased professional competency. Moreover, participants gained more confidence, became more responsible, and had a deeper understanding of oneself. It seems that on account of experiencing the adversity operatives faced their own fragility and weakness, and by overcoming the crisis they became a better version of themselves, gaining a realistic but also challenging attitude towards life (Habib et al., 2018; Morgan et al., 2017). Such process has been well documented by numerous studies (Palmer et al., 2017; Pietrzak et al., 2010).

Changes in interpersonal relationships included resolving conflicts more efficiently, and better sympathizing with other's weakness or pain. Changes in interpersonal relationships presented by Tedeschi and Calhoun (2004) include growth of interpersonal interactions and becoming more intimate with those who were under the same adverse experience. Indeed, participants more apt in resolving conflicts would engage in more interactions with others, leading to a better relationship. Previous qualitative studies of PTG in soldiers have also shown similar results such as a stronger sense of belonging (Habib et al., 2018) and affection for their team (Larick \& Graf, 2012; Scott et al., 2011). Current study results reporting a positive interpersonal change not only in friends or family but also team members support the suggestion that PTG can enhance teamwork (Peterson et al., 2008).

Interestingly, no changes in philosophical and spiritual understandings of life were mentioned by the participants. An adversity or traumatic event experienced by the general public is greatly shocking and stressful due to its unexpectedness (Lowell et al., 2018), and that leads for them to change their life attitude and religious faith (Barrington $\&$ Shakespeare-Finch, 2013; Shaw et al., 2005). However, the event experienced by special forces operatives is highly stressful yet predictable and given by choice, making it difficult to lead to existential contemplation. A participant who had a near-death experience while being dispatched reported that it wasn't considered a traumatic experience because he "did not think any of us would survive during wartime" (Case 1). Because they are trained and mentally prepared for death or injuries during drills or actual missions, adversities may be perceived differently compared to near-death experiences in daily life. These work characteristics may explain the unique experience of operatives in comparison to others suffering from near-death experiences. Another possible explanation is that soldiers tend to rely on personal mental toughness compared to spirituality or religious beliefs 
(Maddi et al., 2006). This could have contributed to the lack of change in philosophical or spiritual viewpoints.

With regards to social changes, most reported positive changes such as recognition and support form co-workers, friends and family members, becoming more united, exchanging practical help and advice, a sense of bonding, and developing comradeship and trust. Special forces operatives recognized that this change had a great impact in enduring and overcoming their adversities. This corresponds to precious studies showing that after an adversity there is an increase in prosocial behavior (Morgan et al., 2011; Putnam, 2002), meaningful interactions (Abrams et al., 2004) and development of a cooperative environment (Didion, 2007; Kaniasty \& Norris, 2004). This shows that special forces operatives can gain psychological benefits through prosocial behavior and overcome adversities even in an environment where people are prone to becoming harsher to each other.

Although it was not a major research question in this study, analysis revealed characteristics associated with overcoming adversities which were divided into personal and environmental factors. Personal factors were related to individual personality, which were lack of self-disclosure regarding hardships, hardiness, a challenging spirit, and being responsible. It can indeed be understood that special forces operatives tend to face and try to overcome adversities rather than avoiding it or seeking alternatives. Environmental factors were characteristics of special forces groups, emphasizing not only hardiness but also horizontal interactions for sharing thoughts and opinions, and acknowledging each other's hardships. These results illustrate that along with personal factors helpful in overcoming adversities and leading to personal growth, it is possible to cultivate an environment that promotes such process. For organizations that inevitably induce adversities or traumatic events due to the nature of their jobs, it seems necessary to help members understand such hardships can be subdued, and cultivate a horizontal organizational culture to induce supportive interactions.

The results of this study have clinical implications in creating pre-emptive interventions to help individuals cope and gain from an adversity. According to Aldwin et al. (1996), whether PTG or psychopathology such as posttraumatic stress disorder occurs depend on how victims manage stress. Similarly, PTG and PGA experienced by special forces operatives after adversities can present new perspectives to those who have developed negative beliefs concerning themselves and the world. For example, those who are experiencing intense negative emotions due to an adversity can start healing by learning that an adversity and trauma are not just painful, but can also lead to a more positive view of oneself and the world, increase challenging spirit, confidence, responsibility, sympathy in others, and conflict resolving skills. In addition, learning that it is necessary to receive help or have meaningful interactions with others rather than endeavoring by themselves can be helpful. In counseling, exploring and confirming positive aspects of the adversity experience can help the reappraisal process for clients.

Given that a steady social network of relationships in the course of adversity can help in overcoming it (Guay et al., 2006), the results also suggest implications regarding the military's communication culture. In general, the military has strict hierarchies and restrained communication, and this culture urges individuals to overcome an adversity by oneself without receiving help (Lynn, 2011). However according to current results, social resources that helped overcome adversities were meaningful communication, pro-social support and social affiliative behaviors, as Mancini (2019) suggested. Therefore, in order to increase resilience and adaptability of military personnel, horizontal communication and meaningful interactions need to be emphasized even in a strictly hierarchical organizational culture by establishing a safe atmosphere to communicate with colleagues.

Research exploring PTG or PGA not only have implications in individual intervention to overcome an adversity and gain personal growth, but also in preventing psychopathology from a community-based, society level. Historically many countries have experienced a variety of national disasters and made great efforts to recover and rebuild from the debris. Global adversities include the earthquake of San Francisco (1906), Halifax explosion (1917), Ohio River flood (1937), 9/11 terror attack (2001) and the recent COVID-19 pandemic. Even in tragic events such as natural disasters individuals can adapt through social support (Calo-Blanco et al., 2017; Hawdon \& Ryan, 2011). Despite the decrease in face-to-face social interactions due to the COVID-19 pandemic, people are currently adapting to the change in environment by utilizing various technology to strengthen social cohesion (Prati \& Mancini, 2021). It is a model example demonstrating that not only the individual's endeavor but the effort of the community can be important in enhancing PGA. In such situations, it is necessary to not only consider individual coping but also what measures are to be made in a social level in order to promote the recovery of its members.

The present study illustrates that participants increased cooperation and social interactions such as intimate conversations, support and advice during adversities. Therefore, nurturing a system that enhances more intimate and constructive interactions among society or community members can be beneficial. Not only at a national level but also for companies and organizations which are going through adversities, establishing an organizational culture promoting cooperation, meaningful interactions and horizontal communication will lay the foundation in overcoming such events.

Although this study has meaningful clinical and social implications, there are limitations in generalizing the results 
due to the unique nature of participants' careers. Since it was the decision of special forces operatives themselves to enlist and go through extreme training in order to be commissioned and pursue a career that includes life-threatening environments, their attitude of coping with adversities may not be typical. Furthermore, the relatively small number of participants due to restrictions of special forces groups can also be a limitation.

It is notable that 21 of the 34 subcategories were variant, meaning that the study was able to capture unique, rich adversities and growth of Korean special forces operatives. However, CQR recommends at least 10 participants for dividing groups into subgroups (e.g., high prosocial group vs low prosocial group) which can enrich the results (Hill, 2012). Since this study had only 8 participants, analysis in distinguishable features among the reported experiences was limited. Lastly, because the focus of this research was not in quantifying the intensity of the adverse event or PTG but the exploration of the personal experience itself, our study did not use quantitative measurement tools (Nguyen et al., 2014; Nivala \& Sarvimäki, 2015). Although exploring unique experiences by utilizing a qualitative research method served as an advantage, we did not use quantitative measurements using a validated scale to objectify their experience.

Further studies regarding adversities need to diversify participants to generalize the reported experience. Exploring subjects with different sex, age, culture, occupation, physical features, family relationships, and diseases will produce more generalizable results leading to stronger clinical and social implications. Furthermore, quantitative studies examining adverse experience with a larger population can shed light upon the relationship in factors related to overcoming adversities and gaining personal growth. This can especially be helpful in managing and preventing negative outcomes of adversities in a national level, by exploring changes society members experienced during and after such events.

Funding Not applicable.

Data and materials availability Applicable.

Code availability Not applicable.

\section{Declarations}

Ethics approval Waived.

Consent to participate Consent with oral and paper form.

Consent for publication Consent with oral and paper form.

Conflicts of interest Not applicable.

\section{References}

Abrams, C. B., Albright, K., \& Panofsky, A. (2004). Contesting the New York community: From liminality to the "new normal" in the wake of September 11. City \& Community, 3(3), 189-220. https:// doi.org/10.1111/j.1535-6841.2004.00078.x

Aldwin, C. M., Sutton, K. J., \& Lachman, M. (1996). The Development of Coping Resources in Adulthood. Journal of Personality, 64(4), 837-871. https://doi.org/10.1111/j.1467-6494.1996.tb00946.x

Andersen, S. B., Karstoft, K.-I., Bertelsen, M., \& Madsen, T. (2014). Original Research Latent Trajectories of Trauma Symptoms and Resilience: The 3-Year Longitudinal Prospective USPER Study. Journal of Clinical Psychiatry, 75(9), 1001-1008.

Arens, A. M. (2015). Trauma management therapy for a veteran with co-occurring combat PTSD and hallucinations: A case study. Clinical Case Studies, 14(2), 115-128. https://doi.org/10.1177/ 1534650114541324

Barrington, A. J., \& Shakespeare-Finch, J. (2013). Working with refugee survivors of torture and trauma: An opportunity for vicarious post-traumatic growth. Counseling Psychology Quarterly, 26(1), 89-105. https://doi.org/10.1080/09515070.2012.727553

Calo-Blanco, A., Kovářík, J., Mengel, F., \& Romero, J. G. (2017). Natural disasters and indicators of social cohesion. PloS one, 12(6), e0176885. https://doi.org/10.1371/journal.pone.0176885

Chappelle, W., Goodman, T., Reardon, L., \& Thompson, W. (2014). An analysis of post-traumatic stress symptoms in United States Air Force drone operators. Journal of Anxiety Disorders, 28(5), 480-487. https://doi.org/10.1016/j.janxdis.2014.05.003

DePrince, A. P., Zurbriggen, E. L., Chu, A. T., \& Smart, L. (2010). Development of the trauma appraisal questionnaire. Journal of Aggression, Maltreatment \& Trauma, 19(3), 275-299. https://doi. org/10.1080/10926771003705072

Dickstein, B. D., McLean, C. P., Mintz, J., Conoscenti, L. M., Steenkamp, M. M., Benson, T. A., Isler, W. C., Peterson, A. L., \& Litz, B. T. (2010). Unit cohesion and PTSD symptom severity in Air Force medical personnel. Military Medicine, 175(7), 482-486. https://doi.org/10.7205/MILMED-D-09-00178

Didion, J. (2007). The Year of Magical Thinking (Reprint). New York: Knopf Doubleday Publishing Group.

Diener, E., \& Seligman, M. E. (2002). Very happy people. Psychological Science, 13(1), 81-84. https://doi.org/10.1111/1467-9280. 00415

Eid, J., \& Morgan, C. A., III. (2006). Dissociation, hardiness, and performance in military cadets participating in survival training. Military Medicine, 171(5), 436-442. https://doi.org/10.7205/ MILMED.171.5.436

Feder, A., Southwick, S. M., Goetz, R. R., Wang, Y., Alonso, A., Smith, B. W., Buchholz, K. R., Waldeck, T., Ameli, R., Moore, J., Hain, R., Charney, D. S., \& Vythilingam, M. (2008). Posttraumatic Growth in Former Vietnam Prisoners of War. Psychiatry: Interpersonal and Biological Processes, 71(4), 359-370. https:// doi.org/10.1521/psyc.2008.71.4.359

Forstmeier, S., Kuwert, P., Spitzer, C., Freyberger, H. J., \& Maercker, A. (2009). Posttraumatic Growth, Social Acknowledgment as Survivors, and Sense of Coherence in Former German Child Soldiers of World War II. The American Journal of Geriatric Psychiatry, 17(12), 1030-1039. https://doi.org/10.1097/JGP.0b013e3181 ab8b36

Greenberg, J., Tsai, J., Southwick, S. M., \& Pietrzak, R. H. (2021). Can military trauma promote psychological growth in combat veterans? Results from the national health and resilience in veterans study. Journal of Affective Disorders, 282, 732-739. https://doi. org/10.1016/j.jad.2020.12.077

Guay, S., Billette, V., \& Marchand, A. (2006). Exploring the links between posttraumatic stress disorder and social support: 
Processes and potential research avenues. Journal of Traumatic Stress, 19(3), 327-338. https://doi.org/10.1002/jts.20124

Habib, A., Stevelink, S., Greenberg, N., \& Williamson, V. (2018). Posttraumatic growth in (ex-) military personnel: Review and qualitative synthesis. Occupational Medicine, 68(9), 617-625. https:// doi.org/10.1093/occmed/kqy 140

Hawdon, J., \& Ryan, J. (2011). Social relations that generate and sustain solidarity after a mass tragedy. Social Forces, 89(4), 13631384. https://doi.org/10.1093/sf/89.4.1363

Hill, C. E. (2012). Consensual qualitative research: A practical resource for investigating social science phenomena. American Psychological Association.

Hill, C. E., Knox, S., Thompson, B. J., Williams, E. N., Hess, S. A., \& Ladany, N. (2005). Consensual qualitative research: An update. Journal of Counseling Psychology, 52(2), 196-205. https://doi. org/10.1037/0022-0167.52.2.196

Hill, C. E., Thompson, B. J., \& Williams, E. N. (1997). A guide to conducting consensual qualitative research. The Counseling Psychologist, 25(4), 517-572. https://doi.org/10.1177/0011000097 254001

Jackson, J. J., Thoemmes, F., Jonkmann, K., Lüdtke, O., \& Trautwein, U. (2012). Military training and personality trait development: Does the military make the man, or does the man make the military? Psychological Science, 23(3), 270-277. https://doi.org/10. 1177/0956797611423545

Kaniasty, K., \& Norris, F. H. (2004). Social support in the aftermath of disasters, catastrophes, and acts of terrorism: Altruistic, overwhelmed, uncertain, antagonistic, and patriotic communities. In R. J. Ursano, A. E. Norwood, \& C. S. Fullerton (Eds.), Bioterrorism: Psychological and public health interventions (pp. 200-229). Cambridge University Press.

Lakey, B., \& Orehek, E. (2011). Relational regulation theory: A new approach to explain the link between perceived social support and mental health. Psychological Review, 118(3), 482-495. https:// doi.org/10.1037/a0023477

Larick, J. G., \& Graf, N. M. (2012). Battlefield Compassion and Posttraumatic Growth in Combat Servicepersons. Journal of Social Work in Disability \& Rehabilitation, 11(4), 219-239. https://doi. org/10.1080/1536710X.2012.730824

Linley, P. A., \& Joseph, S. (2004). Positive change following trauma and adversity: A review. Journal of Traumatic Stress, 17(1), 11-21. https://doi.org/10.1023/B:JOTS.0000014671.27856.7e

Lowell, A., Suarez-Jimenez, B., Helpman, L., Zhu, X., Durosky, A., Hilburn, A., ... Neria, Y. (2018). 9/11-related PTSD among highly exposed populations: A systematic review 15 years after the attack. Psychological Medicine, 48(4), 537-553. https://doi. org/10.1017/S0033291717002033

Lynn, K. H. (2011). The importance of understanding military culture. Social Work in Health Care, 50(1), 4-18. https://doi.org/10.1080/ 00981389.2010.513914

Maddi, S. R., Brow, M., Khoshaba, D. M., \& Vaitkus, M. (2006). Relationship of hardiness and religiousness to depression and anger. Consulting Psychology Journal: Practice and Research, 58(3), 148. https://doi.org/10.1037/1065-9293.58.3.148

Maguen, S., \& Litz, B. T. (2006). Predictors of Morale in US Peacekeepers 1. Journal of Applied Social Psychology, 36(4), 820-836. https://doi.org/10.1111/j.0021-9029.2006.00045.x

Mancini, A. D. (2019). When acute adversity improves psychological health: A social-contextual framework. Psychological Review, 126(4), 486-505. https://doi.org/10.1037/rev0000144

Mancini, A. D., Littleton, H. L., \& Grills, A. E. (2016). Can people benefit from acute stress? Social support, psychological improvement, and resilience after the Virginia Tech campus shootings. Clinical Psychological Science, 4(3), 401-417. https://doi.org/10. 1177/2167702615601001
Mark, K. M., Stevelink, S. A. M., Choi, J., \& Fear, N. T. (2018). Posttraumatic growth in the military: A systematic review. Occupational and Environmental Medicine, 75(12), 904. https://doi.org/ 10.1136/oemed-2018-105166

Mitchell, M. M., Gallaway, M. S., Millikan, A. M., \& Bell, M. R. (2013). Combat exposure, unit cohesion, and demographic characteristics of soldiers reporting posttraumatic growth. Journal of Loss and Trauma, 18(5), 383-395. https://doi.org/10.1080/15325 024.2013.768847

Morgan, G. S., Wisneski, D. C., \& Skitka, L. J. (2011). The expulsion from Disneyland: The social psychological impact of 9/11. American Psychologist, 66(6), 447. https://doi.org/10.1037/a0024772

Morgan, J. K., Desmarais, S. L., Mitchell, R. E., \& Simons-Rudolph, J. M. (2017). Posttraumatic Stress, Posttraumatic Growth, and Satisfaction With Life in Military Veterans. Military Psychology, 29(5), 434-447. https://doi.org/10.1037/mil0000182

Morrow, C. E., Bryan, C. J., Stephenson, J. A., Bryan, A. O., Haskell, J., \& Staal, M. (2013). Posttraumatic stress, depression, and insomnia among US Air Force pararescuemen. Military Psychology, 25(6), 568-576. https://doi.org/10.1037/mil0000021

Mouthaan, J., Euwema, M. C., \& Weerts, J. M. (2005). Band of brothers in UN peacekeeping: Social bonding among Dutch peacekeeping veterans. Military Psychology, 17(2), 101-114. https://doi.org/ 10.1207/s15327876mp1702_3

Nguyen, C. M., Liu, W. M., Phan, T. T., Pittsinger, R., Casper, D., \& Alt, M. (2014). Vietnamese military men's perceptions of the long-term psychological effects of reeducation camps. Psychology of Men \& Masculinity, 15(4), 407. https://doi.org/10.1037/ a0034731

Nivala, S., \& Sarvimäki, A. (2015). The lifelong struggle of Finnish World War II veterans. Aging \& Mental Health, 19(6), 493-499. https://doi.org/10.1080/13607863.2014.954525

Ogle, A. D., \& Young, J. A. (2016). USAF special tactics operator combat exposure and psychological stress. Military Psychology, 28(3), 123-133. https://doi.org/10.1037/mil0000121

Palmer, E., Murphy, D., \& Spencer-Harper, L. (2017). Experience of post-traumatic growth in UK veterans with PTSD: A qualitative study. Journal of the Royal Army Medical Corps, 163(3), 171. https://doi.org/10.1136/jramc-2015-000607

Park, C. L., Cohen, L. H., \& Murch, R. L. (1996). Assessment and Prediction of Stress-Related Growth. Journal of Personality, 64(1), 71-105. https://doi.org/10.1111/j.1467-6494.1996.tb00815.x

Peterson, C., Park, N., Pole, N., D’Andrea, W., \& Seligman, M. E. (2008). Strengths of character and posttraumatic growth. Journal of Traumatic Stress, 21(2), 214-217. https://doi.org/10.1002/jts. 20332

Pietrzak, R. H., Goldstein, M. B., Malley, J. C., Rivers, A. J., Johnson, D. C., Morgan, C. A., \& Southwick, S. M. (2010). Posttraumatic growth in Veterans of Operations Enduring Freedom and Iraqi Freedom. Journal of Affective Disorders, 126(1), 230-235. https:// doi.org/10.1016/j.jad.2010.03.021

Prati, G., \& Mancini, A. D. (2021). The psychological impact of COVID-19 pandemic lockdowns: A review and meta-analysis of longitudinal studies and natural experiments. Psychological Medicine, 51, 201-211. https://doi.org/10.1017/S0033291721000015

Putnam, R. D. (2002). Bowling together. The American Prospect, 13(3), 20-22.

Scott, W. J., McCone, D. R., Sayegh, L., Looney, J. D., \& Jackson, R. J. (2011). Mixed Methods in a Post-Deployment Study of U.S. Army National Guard Soldiers. Journal of Workplace Behavioral Health, 26(4), 275-295. https://doi.org/10.1080/15555240.2011. 618430

Shaw, A., Joseph, S., \& Linley, P. A. (2005). Religion, spirituality, and posttraumatic growth: A Systematic review. Mental Health, Religion \& Culture, 8(1), 1-11. https://doi.org/10.1080/13674 67032000157981 
Special Warfare Command. (2016, August 2). History of Special Warfare Command. https://www.swc.mil.kr:444/swc/998/subview.do.

Sudom, K. A., Lee, J. E., \& Zamorski, M. A. (2014). A longitudinal pilot study of resilience in Canadian military personnel. Stress and Health, 30(5), 377-385. https://doi.org/10.1002/smi.2614

Tedeschi, R. G., \& Calhoun, L. G. (1995). Trauma and transformation: Growth in the aftermath of suffering. Sage.

Tedeschi, R. G., \& Calhoun, L. G. (1996). The posttraumatic growth inventory: Measuring the positive legacy of trauma. Journal of Traumatic Stress, 9(3), 455-471. https://doi.org/10.1007/BF021 03658

Tedeschi, R. G., \& Calhoun, L. G. (2004). Posttraumatic growth: Conceptual foundations and empirical evidence. Psychological Inquiry, 15(1), 1-18. https://doi.org/10.1207/s15327965pli1501_ 01

Tsai, J., El-Gabalawy, R., Sledge, W. H., Southwick, S. M., \& Pietrzak, R. H. (2015). Post-traumatic growth among veterans in the USA:
Results from the National Health and Resilience in Veterans Study. Psychological Medicine, 45(1), 165-179. https://doi.org/ 10.1017/S0033291714001202

Wick, S., \& Nelson Goff, B. S. (2014). A Qualitative Analysis of Military Couples With High and Low Trauma Symptoms and Relationship Distress Levels. Journal of Couple \& Relationship Therapy, 13(1), 63-88. https://doi.org/10.1080/15332691.2014. 865983

Publisher's note Springer Nature remains neutral with regard to jurisdictional claims in published maps and institutional affiliations. 